\title{
Proteasome inhibition sensitizes non-small cell lung cancer to histone deacetylase inhibitor-induced apoptosis through the generation of reactive oxygen species
}

\author{
Chadrick E. Denlinger, MD
}

Brian K. Rundall, DO

David R. Jones, MD

\footnotetext{
From the Department of Thoracic and Cardiovascular Surgery, University of Virginia, Charlottesville, Va.

This study was supported by grants to D.R.J. (NCI CA83920 and the American Association for Cancer Research) and C.E.D. (NCI F32 CA101497).

Read at the Eighty-fourth Annual Meeting of The American Association for Thoracic Surgery, Toronto, Ontario, Canada, April 25-28, 2004.

Received for publication April 24, 2004; revisions received June 18, 2004; accepted for publication July 2, 2004.

Address for reprints: David R. Jones, MD, Department of Surgery, Box 800679, University of Virginia, Charlottesville, VA 22908-0679 (E-mail: djones@ virginia.edu).

J Thorac Cardiovasc Surg 2004;128:740-8

$0022-5223 / \$ 30.00$

Copyright (C) 2004 by The American Association for Thoracic Surgery

doi:10.1016/j.jtcvs.2004.07.010
}

Objectives: The histone deacetylase inhibitor suberoylanilide hydroxamic acid induces apoptosis in some malignancies through mitochondrial injury and generation of reactive oxygen species. Histone deacetylase inhibitors also activate the antiapoptotic transcription factor nuclear factor $\kappa \mathrm{B}$. We hypothesize that proteasome inhibition with bortezomib (Velcade; Millennium Pharmaceuticals, Inc, Cambridge, Mass) will inhibit nuclear factor $\kappa \mathrm{B}$ activation, enhance suberoylanilide hydroxamic acid-induced mitochondrial injury, and sensitize non-small cell lung cancer cells to apoptosis.

Methods: Four tumorigenic non-small cell lung cancer cell lines were treated with nothing, suberoylanilide hydroxamic acid, bortezomib, or both drugs. Nuclear factor $\kappa \mathrm{B}-$ dependent transcription was determined by reporter gene assays and endogenous interleukin 8 transcription. Reactive oxygen species were quantified by using the fluorophore $\mathrm{H}_{2}$ DCFDA. Cell viability was determined on the basis of clonogenic survival, and apoptosis was measured by quantifying caspase- 3 activity and DNA fragmentation. Apoptosis and cell-survival assays were repeated in similarly treated cells incubated in the presence or absence of N-acetyl cysteine. Statistical significance was determined by means of analysis of variance.

Results: Suberoylanilide hydroxamic acid significantly enhanced interleukin 8 and nuclear factor $\kappa \mathrm{B}$-dependent reporter gene transcription, and these effects were inhibited by bortezomib $(P \leq .01)$. Combined treatment with suberoylanilide hydroxamic acid and bortezomib induced greater reactive oxygen species generation, more apoptosis $(P \leq .02)$, and more cell death $(P \leq .001)$ than either drug alone. $\mathrm{N}$-acetyl cysteine diminished the induction of apoptosis and enhanced cell survival $(P \leq .04)$.

Conclusions: Suberoylanilide hydroxamic acid and bortezomib synergistically induce reactive oxygen species generation in non-small cell lung cancer, and this plays a critical role in the induction of apoptosis after treatment. Combined treatment with suberoylanilide hydroxamic acid and bortezomib might be an effective treatment strategy for non-small cell lung cancer.

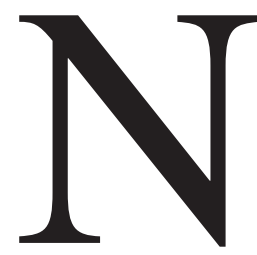
on-small cell lung cancer (NSCLC) remains resistant to chemotherapy-induced apoptosis, despite the advent of thirdgeneration chemotherapeutic agents. ${ }^{1,2}$ Although the molecular targets of different chemotherapies are diverse, the efficacy of all agents depends on exploitation of the endogenous apoptotic machinery. Histone deacetylase inhibitors (HDIs), which induce differentiation, cell-cycle arrest, and apoptosis in some malignancies, are only moderately efficacious in NSCLC, and this relates in part to the activation of the 
antiapoptotic transcription factor nuclear factor $\kappa \mathrm{B}$ (NF$\kappa \mathrm{B}))^{3-5} \mathrm{NF}-\kappa \mathrm{B}$ promotes cell survival after exposure to chemotherapy by driving the transcription of genes that antagonize the apoptotic cascade at multiple levels. ${ }^{6-8}$

Two well-established apoptotic pathways are the extrinsic pathway, which is initiated by ligands binding their respective cell-surface receptors, and the intrinsic pathway, which is mediated through enhanced mitochondrial permeability. ${ }^{9,10}$ Nuclear stress signals resulting from DNA damage converge on the mitochondria, permeabilize the membrane, and allow the subsequent release of apoptosisinducing factor, cytochrome $\mathrm{C}$, and other apoptogenic proteins. ${ }^{11}$ In the presence of adenosine triphosphate, cytochrome $\mathrm{C}$ binds Apaf- 1 and procaspase-9, forming the apoptosome, which activates downstream effecter caspases (caspase-3, caspase-6, and caspase-7), ultimately leading to the morphologic changes of apoptosis. ${ }^{11}$ Suberoylanilide hydroxamic acid (SAHA), an HDI well tolerated in phase I and II clinical trials, has been shown to induce apoptosis through the generation of reactive oxygen species (ROS) and mitochondrial permeabilization. ${ }^{12-14} \mathrm{~A}$ recently delineated mechanism underlying this phenomenon involves acetylation and inactivation of Ku70, the endogenous inhibitor of the BH3-only protein Bax. ${ }^{15}$ Although HDIs induce mitochondrial-mediated apoptosis through Bax disinhibition, they also activate cytoprotective pathways mediated through NF- $\kappa$ B in NSCLC. ${ }^{5}$ Thus simultaneous activation of mutually antagonistic pathways likely contributes to the limited efficacy of HDIs observed in vitro and in vivo in NSCLC. We have recently shown that direct inhibition of $\mathrm{NF}-\kappa \mathrm{B}$ with a dominant negative $\mathrm{I} \kappa \mathrm{B}$, the endogenous NF- $\kappa \mathrm{B}$ inhibitor, dramatically sensitized NSCLC to HDIinduced apoptosis. ${ }^{5}$ Because $\mathrm{I} \kappa \mathrm{B}$ is degraded by proteasomes after phosphorylation and ubiquitination, we subsequently found that the US Food and Drug Administrationapproved proteasome inhibitor bortezomib (Velcade; Millennium Pharmaceuticals, Inc, Cambridge, Mass) also sensitized NSCLC to HDI-induced apoptosis. ${ }^{4}$

Proteasomes are protease complexes comprised of a 20S catalytic core and 2 regulatory 19S subunits. ${ }^{16}$ Proteasomes degrade cytoplasmic and nuclear proteins after ubiquitination and play important roles in regulating eukaryotic cellular processes, such as cell-cycle progression, gene transcription, antigen processing, and degradation of transient signaling molecules, including $\mathrm{I} \kappa \mathrm{B}$ and $\mathrm{Bcl}-2$ family proteins. ${ }^{17-19}$ In addition to inhibiting transcription of anti-apoptotic NF- $\kappa \mathrm{B}$-regulated genes, bortezomib also facilitates accumulation of the proapoptotic proteins Bax, Bad, and Bak, which collectively injure mitochondria and enhance ROS generation. ${ }^{20,21}$ Proteasomes can be inhibited endogenously, and this has been implicated in a self-perpetuating feed-forward cycle leading to apoptosis, in which caspase-3 inhibits proteasome function, leading to subsequent mitochondrial injury and cytochrome C release, which further activates caspase- $3 .^{16}$ Thus normal proteasome function is critical for protecting cells from mitochondrial-mediated apoptosis.

The purpose of this study was to determine whether combined treatment with the HDI SAHA and the proteasome inhibitor bortezomib would induce apoptosis in NSCLC through increased mitochondrial permeability, enhanced generation of ROS, and inhibition of NF- $\kappa \mathrm{B}-$ dependent transcription.

\section{Methods}

\section{Cell-Culture Reagents and Plasmids}

Four tumorigenic NSCLC lines (NCI-H157, NCI-H358, NCIH460, and NCI-A549) obtained from the American Type Culture Collection were cultured in RPMI-1640 (Invitrogen, Carlsbad, Calif) supplemented with $10 \%$ fetal bovine serum (HyClone Laboratories, Logan, Utah) and penicillin-streptomycin. The $3 \mathrm{x}-\kappa \mathrm{B}$ luciferase $(3 \mathrm{x}-\kappa \mathrm{B}$ Luc) reporter construct contains NF- $\kappa$ B DNAbinding consensus sites originally identified in the major histocompatability complex class I promoter and inserted upstream to firefly luciferase. Antibodies against cytochrome $\mathrm{C}$ and $\beta$-tubulin were obtained from Pharmingen (San Diego, Calif) and Sigma (St Louis, Mo), respectively. SAHA was purchased from Biomol (Plymouth Meeting, Pa), bortezomib was kindly provided by Millennium Pharmaceuticals, and N-acetyl cysteine (NAC) was purchased from Calbiochem (La Jolla, Calif).

\section{Cell Viability Assays}

NSCLC cells at 75\% confluency were treated with either nothing or SAHA $(5 \mu \mathrm{mol} / \mathrm{L})$ for $0,24,48$, or 72 hours. In another experiment cells were treated with nothing, SAHA and bortezomib, or SAHA, bortezomib, and NAC concomitantly for 36 hours. Cell viability was then determined by sequentially incubating the cells in $1 \%$ glutaraldehyde and $0.5 \%$ crystal violet for 15 minutes each. The cells were thoroughly washed and dried, and the dye was eluted with Sorenson solution $(30 \mathrm{mmol} / \mathrm{L}$ sodium citrate, $0.02 \mathrm{~mol} / \mathrm{L} \mathrm{HCl}$, and $50 \%$ ethanol) at room temperature for 15 minutes. The optical density of the eluent was measured at a wavelength of $570 \mathrm{~nm}$.

\section{Luciferase Reporter Gene Assays}

NSCLC cells were transfected with $3 \mathrm{x}-\kappa \mathrm{B}$ Luc $(0.5 \mu \mathrm{g} / \mathrm{well})$ with Polyfect (Qiagen, Valencia, Calif), according to the manufacturer's instructions. Six hours after transfection, additional medium was added containing the appropriate pharmacologic agents. After treatment for 18 hours, cells were washed with phosphate-buffered saline and lysed in luciferase reporter buffer (Promega, Madison, Wis). Cells were sequentially snap-frozen at $-80^{\circ} \mathrm{C}$ and thawed at $37^{\circ} \mathrm{C}$. Cell lysates were cleared by means of centrifugation at $13,000 \mathrm{~g}$, and protein concentrations were determined with the Pierce BCA protein assay kit (Pierce, Rockford, Ill). Luciferase assays were performed with the substrate D-Luciferin, and relative light units were measured with an AutoLumat LB953 luminometer (Berthold Analytical Instruments, Pforzheim, Germany). Luminescence was normalized to protein concentrations. 


\section{Western Blotting}

NCSLC cells were treated with nothing, SAHA (5 $\mu \mathrm{mol} / \mathrm{L})$, bortezomib $(50 \mathrm{nmol} / \mathrm{L})$, or both compounds for 24 hours. Mitochondriafree cytoplasmic extracts were prepared by resuspending pelleted cells in 5 pellet volumes of buffer A $(250 \mathrm{mmol} / \mathrm{L}$ sucrose, $20 \mathrm{mmol} / \mathrm{L}$ $\mathrm{N}-2$ =hydroxyethylpiperazine-N-2-ethanesulfonic acid [HEPES], 10 $\mathrm{mmol} / \mathrm{L} \mathrm{KCl}, 1.5 \mathrm{mmol} / \mathrm{L} \mathrm{MgCl}_{2}, 1 \mathrm{mmol} / \mathrm{L}$ ethylenediamine tetraacetic acid, $1 \mathrm{mmol} / \mathrm{L}$ dithiothreitol, and $0.1 \mathrm{mmol} / \mathrm{L}$ phenylmethylsulfonyl fluoride) and incubated on ice for 30 minutes. Cells were homogenized with a Teflon rod before centrifugation at 16,000 $g$ for 30 minutes. Cytoplasmic extracts ( $45 \mu \mathrm{g} / \mathrm{lane})$ were resolved by SDS-PAGE and transferred to nitrocellulose membranes. Primary antibodies against cytochrome $\mathrm{C}$ and $\beta$-tubulin were used for immunoblotting.

\section{Reverse Transcription-Polymerase Chain Reaction}

NCSLC cells were treated for 12 hours under previously described experimental conditions. Cells were lysed with Trizol (Invitrogen), and proteins were extracted with chloroform. RNAs were precipitated with isopropanol and washed with $70 \%$ ethanol. cDNAs were created by using the Advantage RT for PCR enzyme (Clontech, Palo Alto, Calif), and interleukin 8 (IL8) cDNA was amplified by means of PCR with Platinum Taq (Invitrogen) and the primers 5'-CTTCCAAGCTGGCCGTGG-3' and 5' -TGAATTCTCAGCCTTCTT- $3^{\prime}$. As a control, GAPDH cDNA was amplified by using the primers 5'-GTGAGGAGGGGAGATTCAG-3' and 5'-GCATCCTGGGCTACACTG-3'. Polymerase chain reaction products were resolved on a $0.8 \%$ agarose gel.

\section{Apoptosis Assays}

NSCLC cells were plated at $5 \times 10^{5}$ cells per well on 12 -well plates. Twelve hours after plating, cells were either left alone or treated with SAHA, bortezomib, or both drugs. After 24 hours of treatment, cells were harvested, and apoptosis was quantified by means of detection of caspase- 3 activation and DNA fragmentation. Caspase-3 activity was determined by the addition of an APC-DEVD protein conjugate (Calbiochem) to cellular lysates containing $25 \mu \mathrm{g}$ of protein. Fluorescence of caspase-3-cleaved protein conjugates was determined fluorometrically. DNA fragmentation was determined by means of evaluation of cellular nucleosome formation with the Cell Death Detection ELISA Plus kit (Roche, Indianapolis, Ind), according to the manufacturer's instructions.

\section{Measurement of Mitochondrial Membrane Potential and ROS Generation}

Cells were treated for 24 hours with the appropriate agents before incubation with $1 \mathrm{nmol} / \mathrm{L} \quad 3^{\prime} 3^{\prime}$-dihexyloxacarboxyanine iodide $\left(\mathrm{DiOC}_{6}[3]\right.$; Molecular Probes, Eugene, Ore) at $37^{\circ} \mathrm{C}$ for $15 \mathrm{~min}-$ utes and harvesting by means of trypsinization. Cells were washed 3 times with ice-cold phosphate-buffered saline, and mitochondrial membrane potential $\left(\delta \psi_{\mathrm{m}}\right)$ was determined by means of flow cytometry. ROS were measured by incubating trypsinized cells with $\mathrm{H}_{2}$ DCFDA (Molecular Probes) at $22^{\circ} \mathrm{C}$ for 15 minutes in HEPES-buffered saline before analysis by means of flow cytometry.

\section{Colony Formation Assay}

Thinly plated NSCLC cells were treated as described previously. After a 24-hour treatment period, cells were washed, and the drug-containing media was replaced with fresh media. Clonogenic cell survival was determined by allowing cells to grow for an additional week after treatment. Colonies were stained with crystal violet as previously described.

\section{Statistical Analysis and Data Interpretation}

Where appropriate, statistical significance was determined by means of analysis of variance and post-hoc analysis with Bonferroni tests. The study design, data analysis, and interpretation, as well as the decision to submit this article for publication, were not influenced by any outside funding agency.

\section{Results \\ SAHA Induces Minimal Apoptosis and Cell Death in NSCLC}

Despite promising results in preclinical studies with SAHA in prostate and breast cancer, as well as other malignancies, less dramatic results were appreciated in early-phase clinical trials. ${ }^{12}$ To determine whether SAHA induces apoptosis in NSCLC, 4 tumorigenic NSCLC cell lines were treated with a clinically relevant dose of SAHA $(5 \mu \mathrm{mol} / \mathrm{L})$ for 24 hours. ${ }^{12}$ SAHA did not significantly induce apoptosis in any cell line in this short-term apoptosis assay (data not shown). Mitsiades and colleagues ${ }^{22}$ recently indicated that although cells are irreversibly committed to apoptosis within a few hours of exposure to SAHA, execution of apoptosis actually occurs as a significantly delayed event. Therefore to determine whether SAHA induces NSCLC cell death after prolonged exposure, cells was incubated in the presence of SAHA for up to 72 hours. In contrast to previous reports, only marginal diminutions in NSCLC survival were observed after prolonged treatment (data not shown). These data suggest that NSCLC does not respond dramatically to SAHA-induced proapoptotic signals previously demonstrated in other models.

\section{SAHA-Induced Activation of NF- $\kappa$ B Is Suppressed by Bortezomib}

We and others have previously shown that the antiapoptotic transcription factor NF- $\kappa \mathrm{B}$ is a key mediator of the resistance of solid tumors, including NSCLC, to apoptosis. ${ }^{6,7,23}$ SAHA enhanced NF- $\kappa \mathrm{B}$-dependent transcription 8- to 20-fold, as measured by the $3 \mathrm{x}-\kappa \mathrm{B}$ reporter gene assay (Figure $1, A$ ). Because NF- $\kappa$ B inhibition enhances the sensitivity of NSCLC to HDI-induced apoptosis, ${ }^{4,5}$ cells were also treated with bortezomib to determine whether bortezomib could suppress such a dramatic activation of NF- $\kappa$ B. As shown in Figure 1, A, bortezomib suppressed both the basal and SAHA-induced NF- $\kappa \mathrm{B}$ transcriptional activity, as measured by using the transient reporter gene $(P \leq .01)$.

To determine whether SAHA and bortezomib have similar effects on the transcription of endogenous genes, re- 
A.
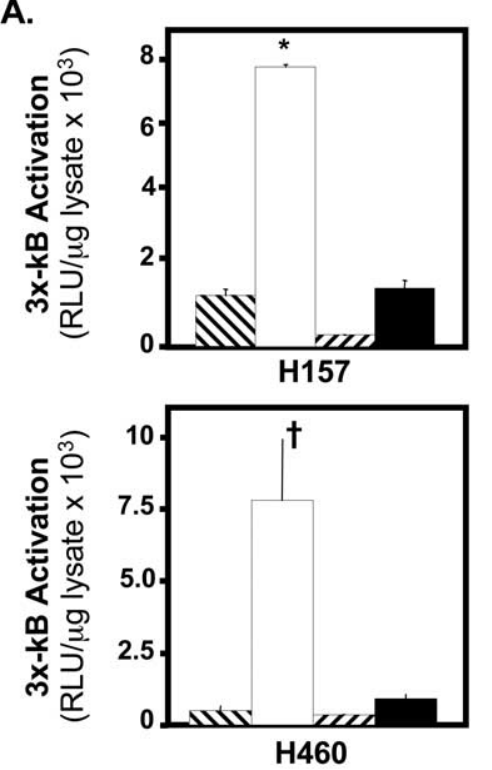
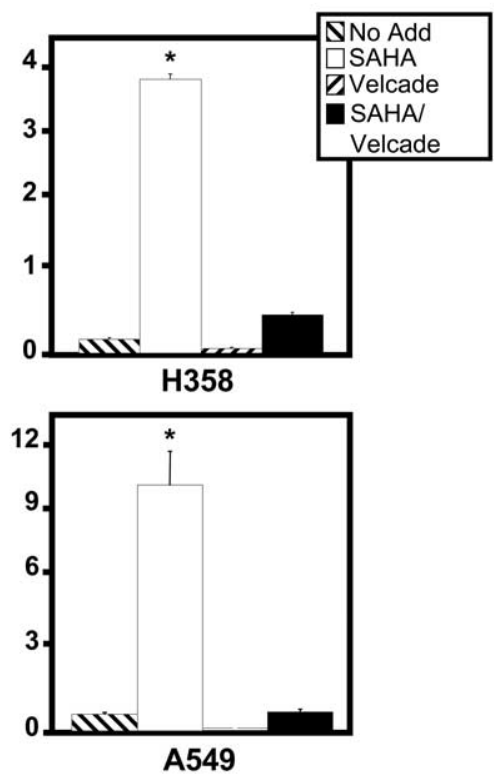

B.

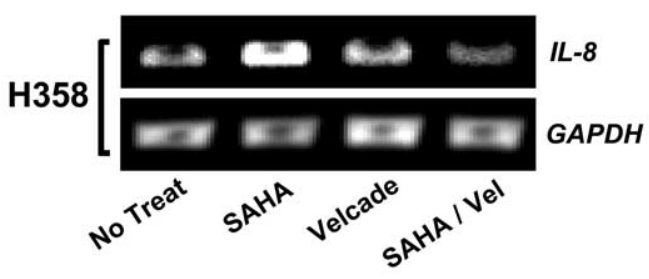

Figure 1. A, NSCLC cells were treated with nothing, SAHA, bortezomib, or both drugs. NF- $\kappa \mathrm{B}-$ dependent transcriptional activity was determined with a transient luciferase reporter gene assay. B, H358 cells were treated as noted in Figure 2, A, and transcription of the NF- $\kappa \mathrm{B}$-dependent gene IL8 was determined by means of RT-PCR. $\dagger P \leq .01$ and ${ }^{*} P \leq .001$, SAHA versus SAHA/bortezomib.

verse transcription-polymerase chain reaction (RT-PCR) analysis was performed on NSCLC cells probing for $I L 8$, a gene tightly regulated by NF- $\kappa$ B. ${ }^{5}$ SAHA dramatically upregulated $I L 8$ transcription, and these effects were attenuated by the addition of bortezomib (Figure 1,B), thus confirming the result seen in Figure 1, A. Collectively, these results demonstrate that SAHA activates NF- $\kappa$ B and that bortezomib suppresses SAHA-induced NF- $\kappa$ B activity to near basal levels.

\section{Bortezomib Sensitizes NSCLC to SAHA-Induced Apoptosis}

Cells were treated for 24 hours with nothing, SAHA, bortezomib, or both drugs to determine whether bortezomib would sensitize NSCLC cells to SAHA-induced death. Neither SAHA nor bortezomib alone significantly induced apoptosis after treatment as single agents. Conversely, combined treatment with both SAHA and bortezomib enhanced DNA frag- mentation $(P \leq .02)$ and caspase-3 activation $(P \leq .01)$ relative to all of the other treatment conditions (Figure 2, $A$ and $B$ ).

To quantify cell death in absolute terms, clonogenic survival assays were performed. As shown in Figure 2, $C$ and $D$, combined treatment with SAHA and bortezomib killed $71 \%$ to $99 \%$ of the cells compared with the other treatment groups $(P \leq .001$; Figure $2, D)$. Because these dramatic results hold promise for further studies in vivo or potentially in phase I clinical trials, subsequent clonogenic survival studies were performed to determine whether concomitant or sequential treatment was more efficacious. A549 cells were treated with SAHA and bortezomib for 24 hours each, and the drugs were administered either sequentially (SAHA followed by bortezomib or in the reverse order) or concomitantly. This study indicated that concomitant therapy was the most efficacious strategy (data not shown). Taken together, these data suggest that the combined therapy of SAHA 
A.

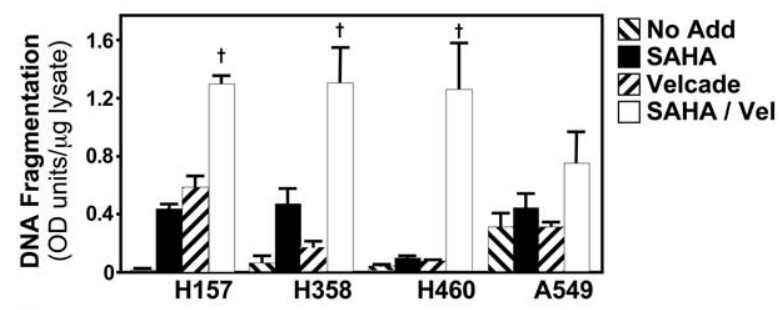

B.

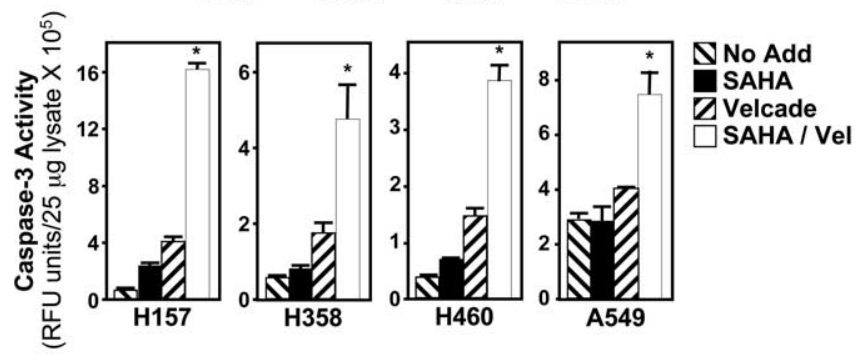

C.

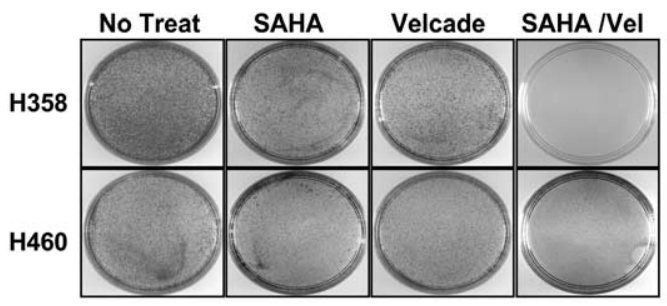

D.

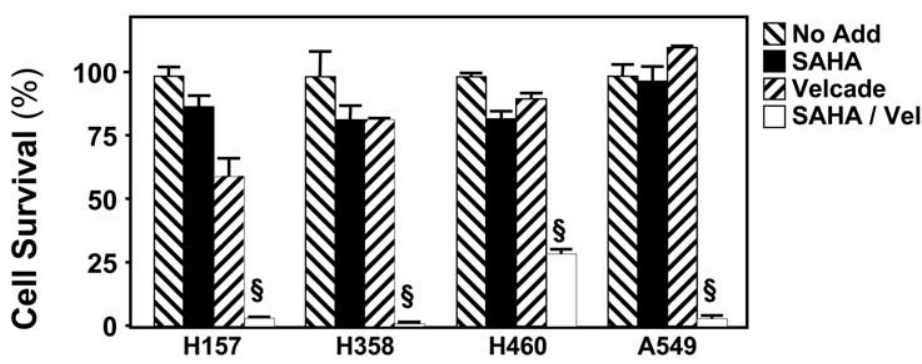

Figure 2. NSCLC cells were treated with nothing, SAHA, bortezomib, or both drugs. Apoptosis was measured by means of DNA fragmentation (A) and caspase-3 activity (B). NSCLC cells were treated with nothing, SAHA, bortezomib, or both drugs, and clonogenic survival assays were performed (C). Colonies were counted and expressed as relative survival compared with that seen in untreated control subjects (D). $+P \leq .02 ;{ }^{*} \leq .01 ; \S P \leq .001$.

and bortezomib significantly enhances apoptosis and cell death in NSCLC and that concomitant administration is the most efficacious strategy in vitro.

\section{SAHA and Bortezomib Induce Mitochondrial Damage and ROS Release}

Because both SAHA and bortezomib have been shown to enhance ROS generation and mitochondrial injury, experiments were performed to address whether combined treatment with SAHA and bortezomib further enhanced ROS generation. As shown in Figure 3, A, isolated treatment with either SAHA or bortezomib minimally enhanced ROS pro- duction in NSCLC. However, combined treatment with both drugs induced more ROS than all other groups.

ROS generation during apoptosis has been associated with loss of $\delta \psi_{\mathrm{m}}{ }^{24}$ Treatment with either SAHA or bortezomib alone slightly increased $\delta \psi_{\mathrm{m}}$. Additionally, combined treatment-enhanced $\delta \psi_{\mathrm{m}}$ did not cause any meaningful $\delta \psi_{\mathrm{m}}$ perturbations beyond what was observed with either drug alone (Figure 3, $B$ ). Similarly, we did not observe any significant changes in $\delta \psi_{\mathrm{m}}$ in any of the treatment conditions when cells were stained with JC-1, an unrelated stain that differentially fluoresces on the basis of the presence of intact $\delta \psi_{\mathrm{m}}$ (data not shown). 
A.

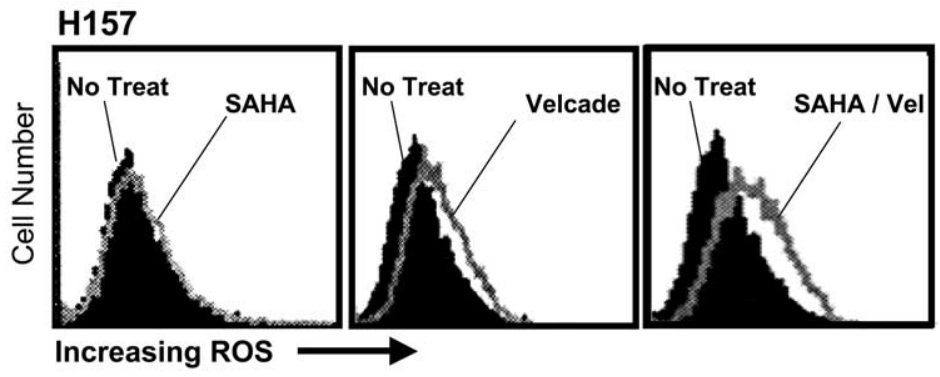

B.
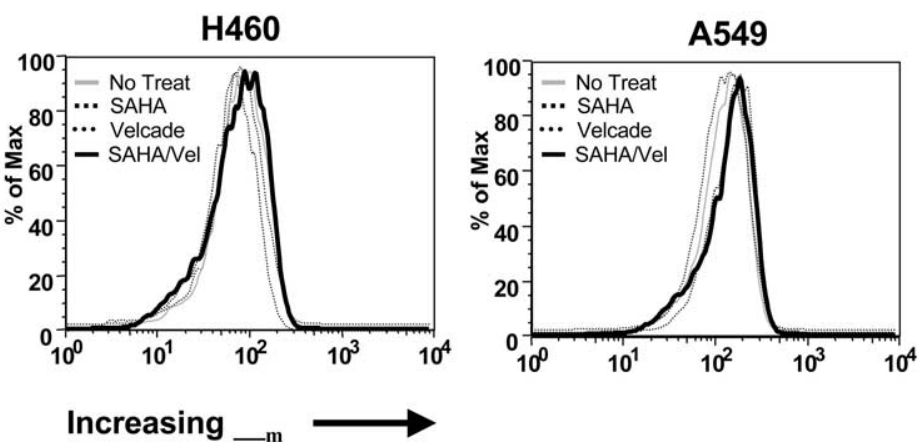

C.
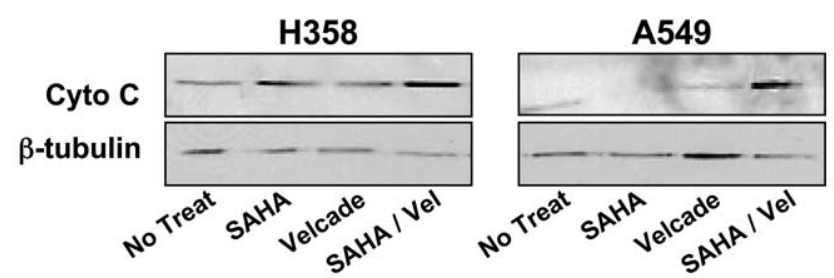

Figure 3. NSCLC cells were treated with nothing, SAHA, bortezomib, or both drugs for 24 hours. A, Presence of ROS was determined by using the ROS-sensitive fluorescent stain $\mathrm{H}_{2}$ DCFDA. $B$, Mitochondrial membrane potential $\left(\delta \psi_{\mathrm{m}}\right)$ was measured by analyzing fluorescent spectral shifts of the $\mathrm{DiOC}_{6}$ stain after treatment. C, Cytochrome C extravasation from the mitochondria was determined by means of Western blot analysis.

Despite the lack of significant changes in $\delta \psi_{\mathrm{m}}$, we next evaluated NSCLC cells for cytochrome C extravasation by means of Western blot analysis in mitochondrial-free cytoplasmic extracts. As shown in Figure 3, C, minimal cytochrome $\mathrm{C}$ was detected in the cytoplasm of untreated cells or cells treated with either SAHA or bortezomib as single agents. However, combined therapy with both SAHA and bortezomib significantly enhanced cytochrome $\mathrm{C}$ extravasation. Therefore the combined treatment synergistically enhanced mitochondrial permeability and cytochrome $\mathrm{C}$ extravasation, despite maintaining a normal electrochemical potential gradient across the mitochondrial membrane.

\section{N-acetyl Cysteine Rescues NSCLC from SAHA/ Bortezomib-Induced Apoptosis}

ROS have previously been described as mediators in the apoptotic cascade, as well as activators of NF- $\kappa \mathrm{B}$, which could explain a potential mechanism by which SAHA activates NF- $\kappa$ B. Experiments were performed to determine whether administration of the free radical scavenger NAC would inhibit SAHA-induced NF- $\kappa \mathrm{B}$ activation and whether NAC could rescue NSCLC from apoptosis induced by SAHA and bortezomib. NSCLC cells were treated with nothing or SAHA in the presence or absence of NAC, and $\mathrm{NF}-\kappa \mathrm{B}-$ dependent transcription was measured with the $3 \mathrm{X}-\kappa \mathrm{B}$ Luc reporter construct. As seen previously, SAHA 
A.

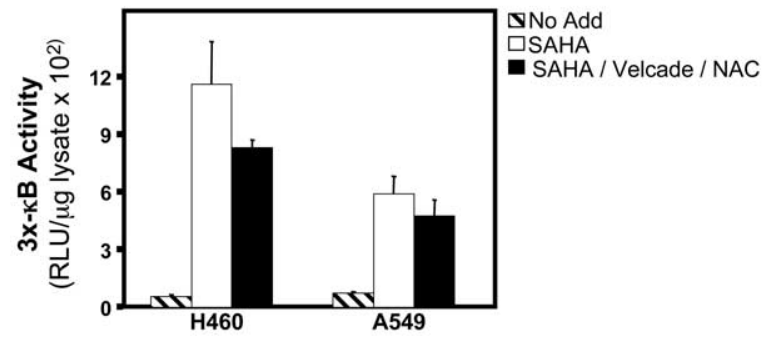

B.

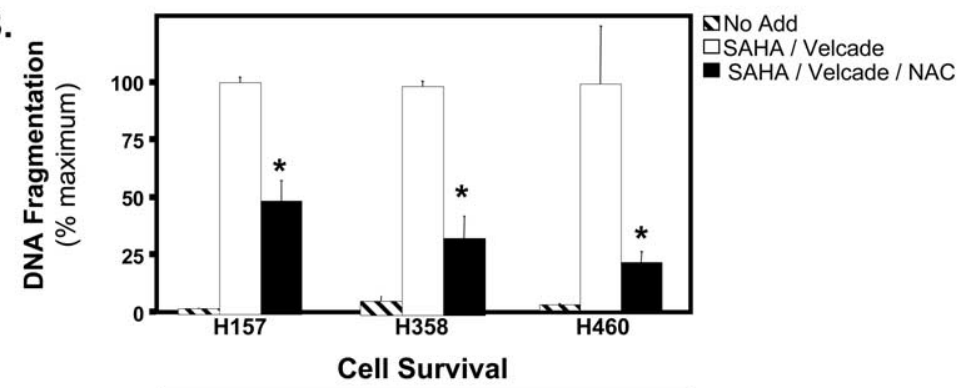

C.

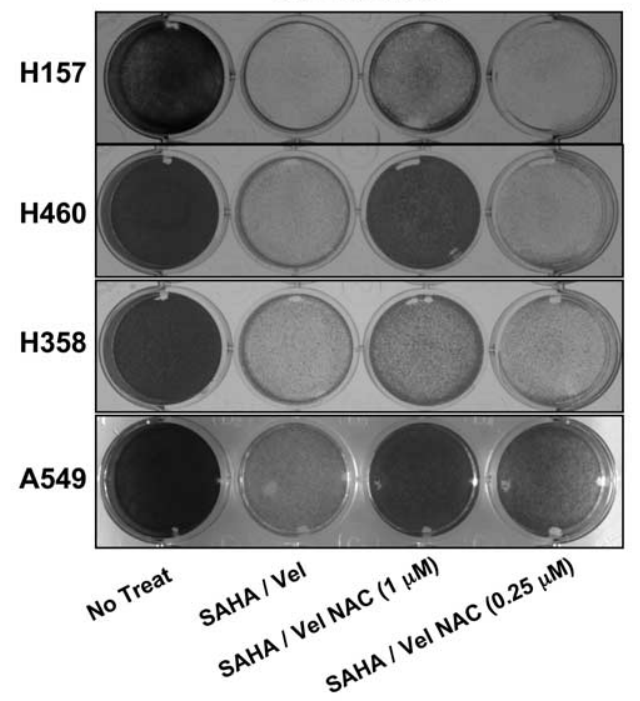

Figure 4. NSCLC cells were treated with nothing, SAHA and bortezomib, or SAHA, bortezomib, and NAC. NF- $\kappa B$-dependent transcription was determined by using the $3 \mathrm{x}-\kappa \mathrm{B}$ reporter gene assay (A). Apoptosis was measured by means of DNA fragmentation (B), and cell survival was determined by staining with crystal violet (C). ${ }^{*} \boldsymbol{P} \leq .04$.

enhanced transcriptional activity of NF- $\kappa \mathrm{B}$, but this was only moderately attenuated by the addition of NAC (Figure 4, A). This suggests that ROS have little or no role in SAHA-induced NF- $\kappa$ B activation.

Cells were treated with nothing, SAHA and bortezomib, or SAHA, bortezomib, and NAC concomitantly to determine whether NAC would rescue NSCLC cells from SAHA/bortezomib-induced apoptosis. NAC significantly diminished apoptosis induced by the combined therapy $(P$ $\leq .04$; Figure $4, B$ ). In a separate experiment cell-survival assays were performed after similar treatment conditions to confirm that changes in apoptosis translated to cell survival. Combined treatment with SAHA and bortezomib nearly eradicated cells; however, NAC enhanced cell survival in a dose-dependent fashion. This suggests that the apoptosis induced by SAHA and bortezomib is dependent, at least in part, on the generation of ROS.

\section{Discussion}

The continued dismal survivals for patients with NSCLC have recently been tempered by the introduction and concomitant enthusiasm for several molecularly targeted treatment strategies. Despite the preclinical promise of these novel agents, the clinical results have been sobering with single therapy alone, regardless of the treatment or its intended molecular target. With improved understanding of 
each tumor's specific biology, there is an increasing appreciation that multiple molecularly targeted therapies might be more efficacious than single-agent therapy.

The data presented in this article build on previous work by our group, ${ }^{4}$ which explores the preclinical merit of combining histone deacetylase and proteasome inhibitors. The genesis of this line of investigation is based on our interest in HDIs, the antiapoptotic transcription factor NF$\kappa \mathrm{B}$, and its role in modulating tumor cell death in NSCLC. There was little cell death induced by single-agent therapy (ie, SAHA) alone, regardless of the length of treatment time. This observation is supported by previous work from our group with other classes of HDIs, including butyrate and trichostatin A. ${ }^{5}$ Although there are several plausible explanations for this, one explanation is that SAHA activates tumor cell-survival signal-transduction pathways, most notably NF- $\kappa$ B. As shown in Figure 1, SAHA induces NF- $\kappa$ B transcriptional activity, and bortezomib completely ameliorates this effect. Moreover, this combined therapy resulted in markedly enhanced apoptotic cell death, as shown in Figure 2. We and others have demonstrated that bortezomib inhibits NF- $\kappa \mathrm{B}$ transcriptional activity and sensitizes NSCLC to chemotherapy and sodium butyrate. ${ }^{4,25}$ Thus it was not surprising to see a similar effect in this model system. It was interesting that there appeared to be more cell death with combined SAHA and bortezomib therapy compared with that seen in our previous study evaluating butyrate and bortezomib. ${ }^{4}$

Several authors have demonstrated that HDI therapy results in appreciable ROS generation. ${ }^{14,21}$ As shown in Figure 3, A, SAHA/bortezomib-induced apoptosis correlated with enhanced ROS generation. In agreement with other studies, ${ }^{14,21}$ we also found that ROS generation was present when either SAHA or bortezomib were used as single agents, although not as much as with combined therapy. ROS have been previously described as critical second messengers for NF- $\kappa \mathrm{B}$ activation. ${ }^{26,27}$ ROS typically activate NF- $\kappa \mathrm{B}$ after receptor mediator-induced nuclear translocation of NF- $\kappa$ B. ${ }^{26}$ In this study ROS did not result in SAHA-induced NF- $\kappa$ B activation given the fact that NAC failed to block SAHA-mediated induction of $\mathrm{NF}-\kappa \mathrm{B}$ (Figure $4, A$ ). In fact, the primary signaling mechanism through which HDIs appear to activate NF- $\kappa \mathrm{B}$ is by enhancing its transactivation potential rather than inducing its nuclear translocation. ${ }^{5}$ Therefore although SAHA did induce low levels of ROS production (Figure 3,A) and activate NF- $\kappa \mathrm{B}$-dependent transcription (Figure 1), these effects appear to be unrelated.

It is interesting that despite the increased production of ROS with this combined treatment strategy, there was preservation of the $\delta \psi_{\mathrm{m}}$. Two predominant but opposing theories on mitochondrial membrane permeability and cytochrome $\mathrm{C}$ extravasation during the induction of apoptosis exist. ${ }^{11}$ One theory, first described in ischemia-reperfusion injury, is based on the development of a permeability transition pore that opens under conditions of oxidative stress, high $\mathrm{Ca}^{2+}$, or low adenosine triphosphate. The open pore allows influx of low-molecularweight solutes, leading to mitochondrial swelling, rupture, and leakage of cytochrome $\mathrm{C}$. The second theory relating to cytochrome $\mathrm{C}$ extravasation during apoptosis contends that Bax and other proapoptotic Bcl-2 member proteins polymerize and insert into the outer mitochondrial membrane and allow cytochrome $\mathrm{C}$ extravasation without disrupting mitochondrial function or the $\delta \psi_{\mathrm{m}}$. These events lead to apoptosis in cells capable of forming the apoptosome but also lead to necrosis in cells not able to form the apoptosome because of the eventual cytochrome $\mathrm{C}$ depletion within the mitochondria. Data presented in this article support the latter theory, in which cytochrome $\mathrm{C}$ extravasation and apoptosis was observed in the absence of significant changes in $\delta \psi_{\mathrm{m}}$ (Figures 2 and 3).

A limitation of this study is that these studies are in vitro experiments, and the results need to be replicated in vivo. However, given the mechanistic nature of this study involving ROS generation and the induction of apoptosis in an ROSdependent manner, repeated studies in vivo would be significantly more difficult to validate this proposed mechanism of action for the combined therapy. Additionally, this study is based on use of a single HDI and a single proteasome inhibitor. Other agents might have resulted in different results. We chose SAHA and bortezomib on the basis of the fact that they represent the most clinically relevant agents currently in their respective drug classes.

In conclusion, this study demonstrates that combined SAHA and bortezomib induces apoptosis in NSCLC and that the interaction between these drugs is mediated in part through the generation of ROS and enhanced mitochondrial permeability. Because both of these agents are being evaluated individually in clinical trials, consideration of combining these compounds in future phase I trials appears warranted.

\section{References}

1. Schiller JH, Harrington D, Belani CP, Langer C, Sandler A, Krook J, et al. The Eastern Cooperative Oncology Group. Comparison of four chemotherapy regimens for advanced non-small-cell lung cancer. N Engl J Med. 2002;346:92-8.

2. Carney DN. Lung cancer-time to move on from chemotherapy. N Engl J Med. 2002;346:126-8.

3. Marks P, Rifkind R, Richon V, Breslow R, Miller T, Kelly WK. Histone deacetylase and cancer. Nat Rev. 2001;1:194-202.

4. Denlinger CE, Keller MD, Mayo MW, Broad RM, Jones DR. Combined histone deacetylation and proteosome inhibition enhances apoptosis in non-small cell lung cancer. J Thorac Cardiovasc Surg. 2004;127:1078-86.

5. Mayo MW, Denlinger CE, Broad RM, Yeung F, Reilly ET, Shi Y, et al. Ineffectiveness of HDAC inhibitors to induce apoptosis involves the transcriptional activation of NF- $\kappa \mathrm{B}$ through the Akt pathway. J Biol Chem. 2003;278:18980-9.

6. Jones DR, Broad RM, Comeau LD, Parsons SJ, Mayo MW. Inhibition of nuclear factor $\kappa \mathrm{B}$ chemosensitizes non-small cell lung cancer through cytochrome c release and caspase activation. $J$ Thorac Cardiovasc Surg. 2002;123:310-7. 
7. Jones DR, Broad RM, Madrid LD, Baldwin AS Jr, Mayo MW Inhibition of $\mathrm{NF}-\kappa \mathrm{B}$ sensitizes non-small cell lung cancer to chemotherapy-induced apoptosis. Ann Thorac Surg. 2000;70:930-7.

8. Denlinger CE, Rundall BK, Jones DR. Modulation of anti-apoptotic signaling pathways in non-small cell lung cancer: the role of NF- $\kappa \mathrm{B}$. Semin Thorac Cardiovasc Surg. 2004;16:28-9.

9. Krueger A, Baumann S, Krammer PH, Kirchhoff S. FLICE-inhibitory proteins: regulators of death receptor-mediated apoptosis. Mol Cell Biol. 2001;21:8247-54.

10. Costantini P, Jacotot E, Decaudin D, Kroemer G. Mitochondrion as a novel target of anticancer chemotherapy. J Natl Cancer Inst. 2000;92: 1042-53.

11. Martinou JC, Green DR. Breaking the mitochondrial barrier. Nat Rev Mol Cell Biol. 2001;2:63-7.

12. Kelly WK, Richon VM, O’Conner O, Curley T, MacGregor-Curtelli B, Tong W, et al. Phase I clinical trial of histone deacetylase inhibitor surberoylanilide hydroxamic acid administered intravenously. Clin Cancer Res. 2003;9:3578-88.

13. Gui CY, Ngo L, Xu WS, Richon WM, Marks PA. Histone deacetylase (HDAC) inhibitor activation of p21WAF1 involves changes in promoterassociated proteins, including HDAC1. Proc Natl Acad Sci U S A. 2004;101:1241-6.

14. Ruefli AA, Ausserlechner MJ, Bernhard D, Sutton VR, Tainton KM, Kefler R, et al. The histone deacetylase inhibitor and chemotherapeutic agent suberoylanilide hydroxamic acid (SAHA) induces a cell-death pathway characterized by cleavage of Bid and production of reactive oxygen species. Proc Natl Acad Sci U S A. 2001; 98:10833-8.

15. Cohen HY, Lavu S, Bitterman KJ, Hekking B, Imahiyerobo TA, Miller $\mathrm{C}$, et al. Acetylation of the $\mathrm{C}$ terminus of $\mathrm{Ku} 70$ by $\mathrm{CBP}$ and PCAF controls Bax-mediated apoptosis. Mol Cell. 2004;13:627-38.

16. Sun XM, Butterworth M, MacFarlane M, Dubiel W, Ciechanover A, Cohen GM. Caspase activation inhibits proteasome function during apoptosis. Mol Cell. 2004;14:81-93.

17. Baumeister W, Walz J, Zuhl F, Seemuller E. The proteasome: paradigm of a self-compartmentalizing protease. Cell. 1998;92:367-90.

18. Li B, Dou QP. Bax degradation by the ubiquitin/proteasome-dependent pathway; involvement in tumor survival and progression. Proc Natl Acad Sci U S A. 2000;97:3850-5.

19. Marshansky V, Wang X, Bertrand R, Luo H, Duguid W, Chinnadurai G, et al. Proteasomes modulate balance among pro-apoptotic and anti-apoptotic Bcl-2 family members and compromise function of the electron transport chain in leukemic cells. J Immunol. 2001;166:3130-42.

20. Pei XY, Grant S. The proteasome inhibitor bortezomib promotes mitochondrial injury and apoptosis by the small molecule Bcl-2 inhibitor HA14-1 in multiple myeloma cells. Leukemia. 2003;17:2036-45.

21. Ling YH, Liebes L, Zou Y, Peres-Soler R. Reactive oxygen specie generation and mitochondrial dysfunction in the apoptotic response to bortezomib, a novel proteasome inhibitor, in human $\mathrm{H} 460$ nonsmall cell lung cancer cells. J Biol Chem. 2003;278:33714-23.

22. Mitsiades CS, Mitsiades NS, McMullan CJ, Poulaki V, Shringarpure R, Hideshima T, et al. Transcriptional signature of histone deacetylase inhibition in multiple myeloma: biological and clinical implications. Proc Natl Acad Sci U S A. 2004;101:540-5.

23. Orlowski RZ, Baldwin AS. NF- $\kappa \mathrm{B}$ as a therapeutic target in cancer. Trends Mol Med. 2002;8:385-9.

24. Li PF, Diets R, Harsdorf R. p53 regulates mitochondrial membrane potential through reactive oxygen species and induces cytochrome Cindependent apoptosis blocked by Bcl-2. EMBO J. 1999;18:6027-36.

25. Denlinger CE, Rundall BK, Keller MD, Jones DR. Proteasome inhibition sensitizes non-small cell lung cancer to gemcitabine-induced apoptosis. Ann Thorac Surg. 2004. In press.

26. Müller JM, Rupec RA, Baeuerle PA. Study of gene regulation by $\mathrm{NF}-\kappa \mathrm{B}$ and $\mathrm{AP}-1$ in response to reactive oxygen intermediates. Methods. 1997;11:301-12.

27. Stall FJT, Roederer M, Leonard A, Herzenberg LA, Herzenberg LA Intracellular thiols regulate activation of nuclear factor $\kappa \mathrm{B}$ and transcription of human immunodeficiency virus. Proc Natl Acad Sci U S A. 1990;87:9943-7.

\section{Discussion}

Dr W. Roy Smythe (Temple, Tex). I enjoyed your talk. As you know, $\mathrm{NF}-\kappa \mathrm{B}$ actually is a transcriptional regulator of a number of genes that all have growth importance in propagation of the neoplastic phenotype. A few years back, in our laboratory, we combined a different proteasome inhibitor with sodium butyrate and saw similar effects, although obviously butyrate is not as clinically relevant as SAHA. But interestingly enough, when we looked at Bcl-XL expression, even though we saw an effect of the proteasome inhibitor on NF- $\kappa \mathrm{B}$, we did not see a compensatory change in Bcl-XL expression, which made the whole paradigm a little bit more questionable.

Did you look at downstream mediators of NF- $\kappa$ B? Did you look at Bcl-XL and phosphatidylinositol 3-kinase, downstream mediators of NF- $\kappa \mathrm{B}$, in this series of experiments?

Dr Denlinger. Yes, we did. We did look at Bcl-xL expression using RT-PCR and found what you found: very little induction by SAHA or, conversely, repression by bortezomib. Therefore at present, we are not certain what downstream effectors we are affecting, despite our findings that combined therapy does result in significant apoptosis.

Dr Dao M. Nguyen (Bethesda, Md). I really enjoyed your talk. I have been following your work in the last few years.

I have a few questions for you. Number one, have you looked at the time course, the kinetic of the ROS generation, and what time after the combination treatment you start seeing the increase in ROS generation?

Dr Denlinger. All of the experiments performed, with the exception of one, were conducted at 24-hour delays after treatment. Therefore we evaluated ROS and cytochrome c extravasation after 24 hours of concomitant treatment with SAHA and bortezomib.

Dr Nguyen. So you think the ROS is coming from mitochondria?

Dr Denlinger. There are conflicting reports in the literature suggesting whether it precedes or follows mitochondrial injury. I think in our model it comes after mitochondrial injury.

Dr Nguyen. Did you try to block the mitochondrial function by overexpressing Bcl-2 to see whether you can abrogate this effect and abrogate the ROS generation, or do you think that the ROS provides a positive feedback loop that keeps on destroying the mitochondria and makes the cells totally committed to death?

Dr Denlinger. We have not tried overexpression of antiapoptotic proteins, such as Bcl-2, Bcl-XL, or Bfl/A1. Again, we believe that ROS generation occurs from mitochondria, but we have not evaluated the feedback loop.

Dr Jack Roth (Houston, Tex). Is this effect selective for cancer cells, or do you also see this in some normal cell populations that you treat with the combination?

Dr Denlinger. We have not evaluated this combination in a noncancerous cell line. We have looked at the combination of sodium butyrate, a different HDI, with bortezomib in animals and saw very little overt toxicity in the mice.

Dr Thomas A. D'Amico (Durham, $N C$ ). Chad, that was beautiful. Is your next step an animal model, and which model will you use?

Dr Denlinger. We would love to continue this study in an animal model, and if we would do this, we would probably generate xenografts with the A549 cell line on the basis of our prior experience. Our limitation currently is gaining access to adequate quantities of the compounds. We prefer using clinically relevant compounds, but the downside of that is that they are often difficult to acquire in adequate supplies to do the in vivo studies. 